\title{
Generation of Acoustic Disturbances in Supersonic Laminar Cavity Flows
}

\author{
Weipeng Li \\ Dept. of Aeronautics and Astronautics, Shanghai Jiaotong University, Shanghai, 200-240, China
}

Taku Nonomura and Kozo Fujii

Institute of Space and Astronautics Science/JAXA, Sagamihara, Kanagawa, 252-5210, Japan

\begin{abstract}
(Received 13 May 2012; accepted 20 October 2014)
The generation of acoustic disturbances in supersonic laminar cavity flows is investigated by large-eddy simulations of supersonic laminar flow $(M=1.2,2.0$, and 3.0) past a rectangular cavity with a length-to-depth ratio of 2. Results suggest that well-originated large-scale vortical structures with strong spanwise coherence are present in the shear layer. Compressibility effects have significant impacts on the shear-layer development and the fluctuation properties. The dominant mechanism for the acoustic radiation in supersonic laminar cavity flows is shown to be associated with the successive passage of large-scale vortices over the cavity trailing edge. It is found that Mach waves radiated from the cavity shear layer may have significant contributions for the noiseradiation in terms of enhancing the strength of the feedback compression waves.
\end{abstract}

\section{INTRODUCTION}

Supersonic flow past cavities has been studied for many years, both in practical and academic interest. In general, when the length-to-depth ratio $(L / D)$ of a rectangular cavity is less than ten, the shear layer reattaches on the cavity aft wall. This type of cavity flow is termed open cavity flow. ${ }^{1,2}$ An undesirable problem in open cavity flow is the existence of intense resonant noise. For instance, a resonance with a sound pressure level of 160 decibels is observed in a cavity flow at Mach number $2.0 .^{3}$ The resonant noise may harm the nearby equipment and the environment, for example, by causing structural vibrations and fatigue, adverse effects on store separation, and undesirable noise.

Reviews of cavity flows were conducted by Grace, Colonius, Rockwell and Naudascher, ${ }^{4}$ and Lawson and Barakos. ${ }^{5}$ The driving mechanism of cavity oscillations is widely regarded as a feedback mechanism between shear-layer instabilities and acoustic disturbances. It was first proposed by Powell for the study of edge tones. ${ }^{6}$ In 1964, Rossiter developed a semiempirical formula to predict the resonance frequencies based on his extensive experimental data with freestream Mach number within the range of 0.5 and 1.2. ${ }^{7}$ Heller et al. improved Rossiters formula by introducing a temperature recover factor. ${ }^{8}$ A typical feedback cycle consists of four key procedures: (1) the shear layer near the leading edge of the cavity is excited by acoustic disturbances, which leads to the generation of discrete vortices; (2) the vortices grow large as they convect downstream and eventually impact on the cavity trailing edge; (3) feedback compression waves (upstream-traveling) are radiated near the cavity trailing edge; and (4) the feedback compression waves propagate upstream and excite the shear layer again. Then, the feedback cycle is closed.

Despite the fact that the feedback mechanism itself has been well established and accepted, the mechanism driving the selfsustained oscillations in supersonic cavity flows is still not pre- cisely resolved. One of the most important factors is the generation of acoustic disturbances near the downstream cavity lip. Rossiter observed that discrete vortices are shed periodically from the leading edge of the cavity and convect downstream until they encounter the downstream cavity lip. ${ }^{7}$ He assumed that the passage of vortices over the trailing edge is responsible for the acoustic radiation. Heller and Bliss emphasized that discrete vortices were not usually observed in their experiment with a Mach number varying from 0.8 to $2.0{ }^{9}$ They stated that the generation of acoustic disturbances is caused by the periodic mass addition and removal near the cavity trailing edge. Zhang reported that the compression wave emission is related to the shear layer deflection, which, in turn, is associated with the vortex production and convection. ${ }^{10}$ By description of an oscillation cycle, Tam et al. stated that the upstream-travelling compression wave is generated by a pressure wave reflection at the bottom aft wall. ${ }^{11}$ An experimental study by Schmit et al. showed that the entrained waves are the start of the feedback loop process rather than the shear layer impingement on the downstream cavity wall, as many references have indicated. ${ }^{12}$ Supersonic laminar flow past cavities usually exists over the orbiter launch/reentry trajectories; however, limited investigations have been conducted. Krishnamurty experimentally observed that the laminar cavity flow produced louder resonant noise than that of turbulent cavity flow. ${ }^{13}$ Heller et al. reported that no resonance was observed in the turbulent cavity flow at Ma3.0; however, a strong resonant peak occurred in the laminar cavity flow. ${ }^{14}$ Based on large-eddy simulations of high subsonic laminar cavity flow, Gloerfelt et al. stated that the strong unsteadiness of the internal recirculation flow can be associated with the possible vortex coalescence. ${ }^{15}$ The physical mechanisms underlying the self-sustained oscillations in supersonic laminar cavity flows are in need of more study, especially on the generation of acoustic disturbances.

This paper aims to address the generation mechanism of 